\title{
Vulvar Alveolar Rhabdomyosarcoma
}

National Cancer Institute

\section{Source}

National Cancer Institute. Vulvar Alveolar Rhabdomyosarcoma. NCI Thesaurus. Code C128273.

An extremely rare malignant mesenchymal neoplasm of the vulva exhibiting skeletal muscle differentiation with an alveolar pattern. 